\title{
Tunable terahertz broadband absorber based on a composite structure of graphene
} multilayer and silicon strip array

\author{
Zhai, Zhaocheng; Zhang, Le; Li, Xiangjun; Xiao, Sanshui
}

Published in:

Optics Communications

Link to article, DOI:

10.1016/j.optcom.2018.09.017

Publication date:

2019

Document Version

Peer reviewed version

Link back to DTU Orbit

Citation (APA):

Zhai, Z., Zhang, L., Li, X., \& Xiao, S. (2019). Tunable terahertz broadband absorber based on a composite structure of graphene multilayer and silicon strip array. Optics Communications, 431, 199-202.

https://doi.org/10.1016/j.optcom.2018.09.017

\section{General rights}

Copyright and moral rights for the publications made accessible in the public portal are retained by the authors and/or other copyright owners and it is a condition of accessing publications that users recognise and abide by the legal requirements associated with these rights.

- Users may download and print one copy of any publication from the public portal for the purpose of private study or research.

- You may not further distribute the material or use it for any profit-making activity or commercial gain

- You may freely distribute the URL identifying the publication in the public portal 


\title{
Tunable terahertz broadband absorber based on a composite structure of graphene multilayer and silicon strip array
}

\author{
Zhaocheng Zhai ${ }^{1}$, Le Zhang* ${ }^{* 1,2}$, Xiangjun $\mathrm{Li}^{1}$, Sanshui Xiao ${ }^{2}$ \\ ${ }^{1}$ Centre for THz Research, China Jiliang University, Hangzhou 310018, China \\ ${ }^{2}$ DTU Fotonik, Department of Photonics Engineering, Technical University of Denmark, Kongens \\ Lyngby, DK-2800, Denmark \\ *zhangle@cjlu.edu.cn
}

keywords:

Terahertz wave

broadband absorber

tunable absorber

multilayer graphene

\begin{abstract}
We propose a terahertz broadband absorber composed by silicon strips with continuous graphene on top of a metal mirror. The simulation results show that under the combined effect of the Fabry-Perot resonance and the dipole mode oscillation excited in the silicon strip array interacting with graphene, this structure can achieve an ultra-wide absorption band from 0.73 to $1.95 \mathrm{THz}$ with absorbance of $90 \%$. By changing the size of the silicon strips or the Fermi level of graphene, the working band of the absorber can be tuned.
\end{abstract}

\section{Introduction}

Terahertz (THz) wave has many applications for biomedical security check, sensing and wireless communication ${ }^{[1-5]}$. With the rapid development of $\mathrm{THz}$ science and technology, more functional devices composed of different materials and structures are required to meet more efficient and convenient demands. In recent years, graphene, a monolayer of carbon atom, has found an important position in the fields of electronics, optoelectronics, thermals and mechanics because of its unique physical properties ${ }^{[6-9]}$. Graphene supports surface plasmon polarization in $\mathrm{THz}$ range. The surface conductivity of graphene depends on its chemical potential which can be controlled by gate voltage, magnetic field, electrostatic doping as well as optical excitation ${ }^{[10-12]}$. As a result, graphene can be used for designing $\mathrm{THz}$ functional devices such as filters ${ }^{[13]}$, reflectors $^{[14]}$, modulators ${ }^{[15]}$, absorbers ${ }^{[16-29]}$ and polarizers ${ }^{[30]}$. Focusing on $\mathrm{THz}$ absorbers, there are also various reported types including dual-frequency ${ }^{[16,17]}$, multifrequency $^{[18]}$, broadband ${ }^{[19-21]}$ or tunable absorption ${ }^{[22,23]}$. Arya Fallahi et al. first proposed several graphene metasurfaces that can be used to dynamically control the absorption, reflection or polarization of electromagnetic wave ${ }^{[19]}$. Andryieuski et al. achieved both narrowband and broadband dynamic tunable absorption in the THz band by graphene metamaterials ${ }^{[20]}$. Yin Zhang et al. use a two-layer graphene grid and a cross-metal subwavelength structure to obtain narrow-band THz absorber with dynamic tunable absorption and polarization independence ${ }^{[21]}$. More publications by Xiong et al. ${ }^{[22]}$, Rahmanzadeh et al. ${ }^{[23,24]}$, Zou et al. ${ }^{[25]}$, Xu et al. ${ }^{[26]}$, Fu et al. ${ }^{[27]}$, Fardoost et al. ${ }^{[28]}$, 
Torabi et al. ${ }^{[29]}$ reported a number of absorbers based on graphene metamaterials. Most of these reported designs use multiple layers of patterned graphene and different graphene layer may have different absorption resonance. When multiple layers are stacked, each absorption resonance is superimposed on the absorption spectrum thus broadening the absorption band. With graphene metasurfaces the structure becomes relatively complicated and less easy for fabrication.

In this paper, a THz absorber consisting of silicon strip array, unpatterned graphene layer, polymer layer and metal plate is proposed. Its working bandwidth and absorbance was numerically studied. The dipole mode oscillation in the silicon strip array broadens the original absorption peak of graphene layer. The performance was optimized by adjusting the size of the silicon strip and the Fermi level of graphene. Simulation shows although this absorber has no complicated structure such as patterned graphene, it still provides broadband absorption in $\mathrm{THz}$ band.

\section{Theoretical model}

The schematic of the proposed absorber is shown in Fig.1(a). The structure has two-dimensional periodicity in x-y plane with the period $p$. From top to bottom, each unit cell consists of four silicon strips, multilayer graphene, a TOPAS polymer substrate and a bottom metal plate mirror. The length and width of each silicon strip are $l$ and $w$ satisfying $l+2 w=p$. The thickness of silicon strip is $h_{\text {s }}$ while that of polymer layer is $d$. The surface conductivity of graphene depends on its Fermi level. The interband conductivity of graphene is much lower than the intraband one in $\mathrm{THz}$ range. Thus, the conductivity of monolayer graphene can be approximated by the Drude model ${ }^{[31]}$ :

$$
\sigma(\omega)=\frac{\mathrm{e}^{2} E_{\mathrm{f}}}{\pi \hbar^{2}} \frac{\mathrm{i}}{\omega+\mathrm{i} \tau^{-1}}
$$

where $\hbar$ is the reduced Planck's constant, $e$ is the elementary charge, $\omega$ is the angular frequency, $E_{\mathrm{f}}$ is the energy of Fermi level and $\tau$ is the carrier relaxation lifetime. The conductivity has a minimum value of $\sigma_{\min }=\mathrm{e}^{2} / 4 \hbar$ with zero Fermi level.

(a)

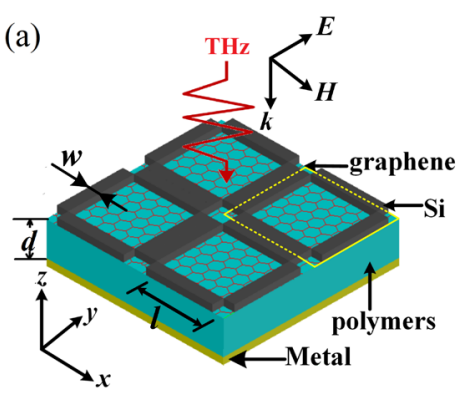

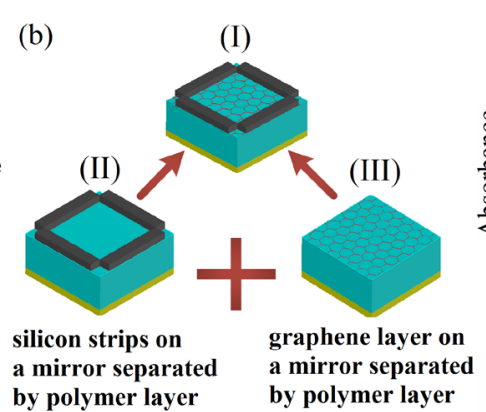

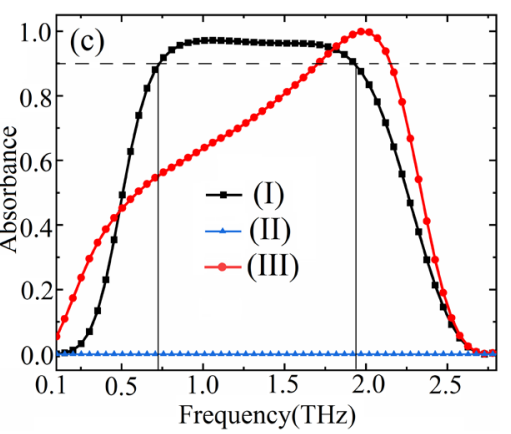

Fig. 1. (a) The schematic of the proposed structure; (b) Schematic diagram of the original structure (I), a reduced structure (II) with only silicon strips on a mirror separated by lossless polymer layer, another reduced structure (III) with only graphene on a mirror separated by lossless polymer; (c) The absorbance spectra of the structure (I) (black line), (II) (blue line) and (III) (red line).

Numerical simulation is conducted by using finite-difference time-domain(FDTD) software package (Lumerical FDTD solutions). As shown in Fig.1(a), THz wave is normally incident into the device along $\mathrm{z}$-axis with its electric field in $\mathrm{x}-\mathrm{y}$ plane. Periodic 
boundary conditions are used in the $\mathrm{x}$ and $\mathrm{y}$ directions, and perfectly matched layer (PML) boundary conditions are used in the $\mathrm{z}$ directions. The power absorbance is expressed as $A=1-R-T$ under the condition of normal incidence, where $R$ is the reflectance and $T$ is the transmittance. Since the skin depth of $\mathrm{THz}$ wave on the metal sheet is in the order of nanometers, the transmittance is very close to zero. So the absorbance can be simplified as $A=1-R$.

\section{Results and Discussion}

The parameters of our designed structure are chosen as follows: $p=32 \mu \mathrm{m}, l=24 \mu \mathrm{m}$, $w=4 \mu \mathrm{m}, d=35 \mu \mathrm{m}, h_{\mathrm{s}}=1 \mu \mathrm{m}$. The refractive indices of high resistance silicon and TOPAS polymer are $n_{\mathrm{s}}=3.45$ and $n_{\mathrm{t}}=1.53$ respectively while their material loss and dispersion are neglected. Here, two layers of continuous graphene with the Fermi energy $E_{\mathrm{f}}=0.3 \mathrm{eV}$ and carrier relaxation lifetime $\tau=1.25 \mathrm{ps}$ are used between the silicon array and the polymer substrate. The purpose of using two layers here is to get sufficient conductivity under a small Fermi energy, since multilayer graphene means multiplying effective surface conductivity. In practice, this can be realized by transferring monolayer graphene onto polymer substrate twice. The transferred graphene is usually hole-doped with a Fermi level of approximately $-0.3 \mathrm{eV}^{[32]}$, which is enough for the design in this paper so that we do not need to electrostatically tune the Fermi level of graphene.

The black curve in Fig. 1(c) illustrates the absorbance spectrum from $0.1 \mathrm{THz}$ to $2.8 \mathrm{THz}$ of the original designed absorber structure (illustrated as (I) in Fig. 1(b)), which shows structure (I) can achieve an absorption bandwidth of $1.22 \mathrm{THz}$ from $0.73 \mathrm{THz}$ to $1.95 \mathrm{THz}$ with absorbance larger than $90 \%$. The dashed line at absorbance $=0.9$ presents the threshold of the absorption working band. To study the mechanism of the absorber, we calculated the absorption spectrum of a reduced structure with only silicon strips on a mirror separated by polymer layer (illustrated as (II) in Fig.1 (b)). Its absorbance in blue line of Fig. 1(c) shows that structure (II) cannot absorb $\mathrm{THz}$ waves because they are just totally reflected by the bottom metal mirror. Moreover, we calculated the absorption spectrum of another reduced structure with only graphene on a mirror separated by polymer (shown as (III) in Fig.1(b)). There is a relatively narrow absorption peak around $1.9 \mathrm{THz}$ in the absorbance spectrum of structure (III) in red line of Fig. 1(c). Thus structure (III) only achieves a narrow absorption band from $1.71 \mathrm{THz}$ to $2.14 \mathrm{THz}$. When the two reduced structures are combined into the original one (I), the absorption band is much broadened. The reason can be explained from the electric field distribution of the structure in $\mathrm{x}-\mathrm{y}$ plane and $\mathrm{y}-\mathrm{z}$ plane in Fig.2. We choose three frequency points of $0.4 \mathrm{THz}, 0.9 \mathrm{THz}$ and $1.9 \mathrm{THz}$ while the first one is out of the absorption band (with absorbance $>90 \%$ ), the second locates among lower frequencies inside the absorption band and the third locates among higher frequencies inside the band. Fig.2(a) and (d) shows the structure can confine part of the incident wave at 0.4 $\mathrm{THz}$ out of working band, thus the absorbance is moderate. However, around $0.9 \mathrm{THz}$ the wave localization is more obvious at the interface between silicon and graphene as shown in Fig.2(b) and (e). This is due to the free-space electromagnetic wave couples to the dipole mode in the silicon strip array and the oscillation is enhanced interacting with graphene. From Fig.2(c) and (f), the major part of the electromagnetic energy is localized between the graphene and the metal mirror near 1.9 THz. Graphene multilayer, polymer layer and metal plate form the Fabry-Perot (FP) resonator. The multiple reflection happens in the FP resonator and the reflected waves coherently cancel out each other, leading to enhanced absorption in the cavity. As a result, both the 
dipole mode in the silicon strip array and the FP resonant enhancement in graphenebased multilayer leads to the broad absorption band of structure (I).

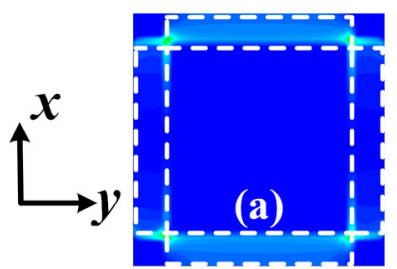

$0.4 \mathrm{THz}$

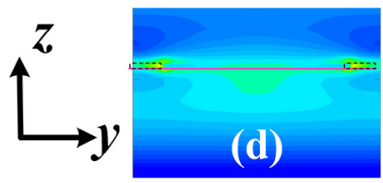

$0.4 \mathrm{THz}$

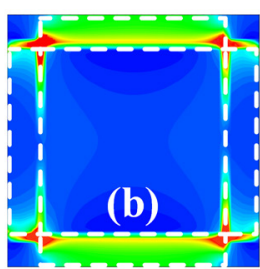

$0.9 \mathrm{THz}$

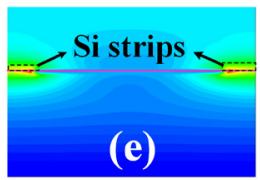

$0.9 \mathrm{THz}$

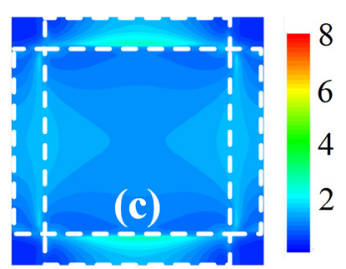

$1.9 \mathrm{THz}$

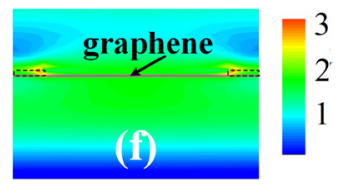

$1.9 \mathrm{THz}$

Fig. 2. The electric field distribution in $x-y$ plane $(z=0)$ at (a) $0.4 \mathrm{THz}$, (b) $0.9 \mathrm{THz}$, (c) $1.9 \mathrm{THz}$ and the electric field distribution in $y-z$ plane $(\mathrm{x}=0)$ at (d) $0.4 \mathrm{THz}$, (e) $0.9 \mathrm{THz}$, (f) $1.9 \mathrm{THz}$.
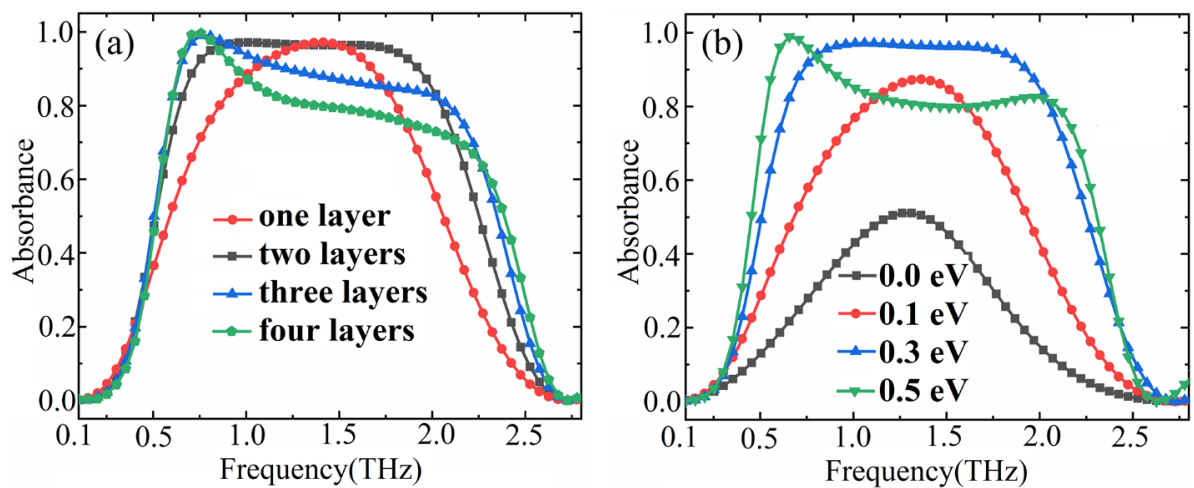

Fig. 3. (a) The absorbance spectra of the structure with one, two, three and four layers graphene when $E_{\mathrm{f}}=0.3 \mathrm{eV}$; (b)The absorbance spectra under different Fermi level $E_{\mathrm{f}}$ of two-layer graphene.

For the discussion above, we considered two-layer graphene in the structure. Fig.3(a) showing the spectra of the absorber with different layers of graphene when $E_{\mathrm{f}}=0.3 \mathrm{eV}$. If we use one, three or four layers, the absorption band is narrowed. This can be explained by a compensate problem if using an equivalent circuit model ${ }^{[20]}$. The proposed multilayer structure can be equivalent to a transmission line circuit and graphene multilayer can be equivalent to the conductivities in the circuit. The absorption of the circuit highly depends on the value of the conductivities and do not follows the higher conductivity, the larger absorption. Since multilayer graphene means multiplying effective surface conductivity, only a suitable conductivity in match with the circuit condition will lead a perfect absorption. The absorption spectra under different graphene Fermi level $E_{\mathrm{f}}$ shown in Fig. 3(b) can also be understood with similar explanation. When $E_{\mathrm{f}}$ is lower than $0.1 \mathrm{eV}$, the maximum absorbance reduces to $88.2 \%$. Due to the high value of real conductivity, the absorbance is only $52.3 \%$ at $1.25 \mathrm{THz}$ when the Fermi level is $0 \mathrm{eV}$. The absorbance is decreasing when the Fermi level increases to $0.5 \mathrm{eV}$ also because of the change of the conductivity. When $E_{\mathrm{f}}$ is about $0.3 \mathrm{eV}$, the conductivity of two-layer graphene is best suitable for the equivalent circuit to get maximum absorbance thus the absorber obtains optimum performance. The maximum absorbance reaches $97.7 \%$. In addition, two-layer graphene in this 
design can be substituted with monolayer graphene. However, to keep the absorption performance, we need to increase $E_{\mathrm{f}}$ to more than twice the original value. Under this situation, the graphene layer need to be electrostatically tuned in practical use.

To confirm the advantage of the designed absorber, it is necessary to compare our results with previously reported graphene-based $\mathrm{THz}$ absorbers (see Table 1). Fractional bandwidth $(\mathrm{BW})$ is defined as $\alpha=\frac{\Delta f}{f_{\text {center }}}$, where $\Delta f$ is the bandwidth and $f_{\text {center }}$ is the center frequency of the absorption working band. The absorber in this paper, which is based on continuous graphene, achieves an absorption BW of $1.22 \mathrm{THz}$ from $0.73 \mathrm{THz}$ to $1.95 \mathrm{THz}$ and the fractional $\mathrm{BW}$ is $91 \%$. The comparison shows although our fractional BW is not broadest, the absorber in this paper can still be considered as broadband under the condition that the structure is relatively simple with continues graphene. The fabrication may thus be easier for practical $\mathrm{THz}$ applications. Also the mechanism is not the same as those designs using multiple layers of patterned graphene, in which the absorption resonance of each layer is superimposed on the absorption spectrum to broaden the absorption band. The absorption band in this paper results from the original absorption peak of graphene layer broadened by the dipole mode oscillation in the silicon strip array.

Table 1. Comparison of the absorber in this paper with published graphene-based THz absorbers

\begin{tabular}{llll}
\hline Reference & $\begin{array}{l}\text { Absorption } \\
\text { Band (THz) }\end{array}$ & $\begin{array}{l}\text { Fractional } \\
\text { BW (\%) }\end{array}$ & Graphene type \\
\hline$[24]$ & $0.55-3.12$ & 140 & $\begin{array}{l}\text { Multiple graphene layers with circle, cross and } \\
\text { square shape }\end{array}$ \\
\hline$[26]$ & $3-7.8$ & 88.9 & $\begin{array}{l}\text { Multiple graphene ribbon layers with different } \\
\text { widths }\end{array}$ \\
\hline$[27]$ & $5.5-7.1$ & 25.3 & $\begin{array}{l}\text { Multiple graphene layers including graphene ring } \\
\text { and two "T" shaped graphene bands }\end{array}$ \\
\hline$[28]$ & $1.65-4.35$ & 90 & $\begin{array}{l}\text { Multiple graphene layers with fishnet graphene } \\
\text { unit cell }\end{array}$ \\
\hline$[29]$ & $2-4$ & $\begin{array}{l}\text { Irregular patterned graphene frequency selective } \\
\text { surface }\end{array}$ \\
\hline
\end{tabular}
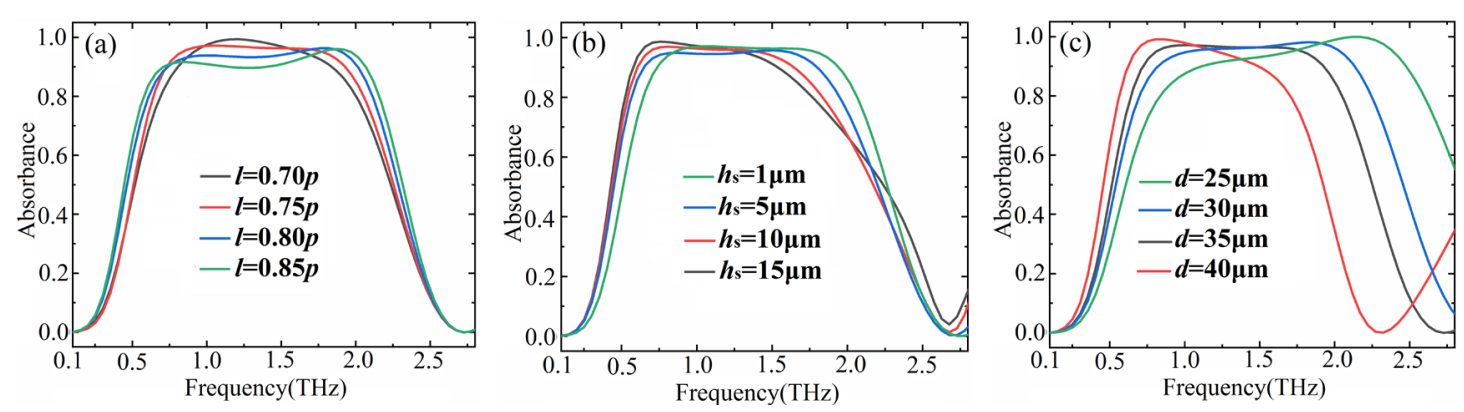

Fig.4. The absorbance spectra under (a) different strip length $\boldsymbol{l}$ when $d=35 \mu \mathrm{m}, h_{\mathrm{s}}=1 \mu \mathrm{m}$; (b) different strip height $h_{\mathrm{s}}$ when $d=32 \mu \mathrm{m}, l=24 \mu \mathrm{m}$; (c) different polymer thickness $d$ when $l=24 \mu \mathrm{m}, h_{\mathrm{s}}=1 \mu \mathrm{m}$.

At last, for optimization purpose, we tuned the working band of the absorber by changing the length $l$, height $h_{\mathrm{s}}$ of silicon strips and thickness $d$ of the polymer substrate 
when fixing the graphene $E_{\mathrm{f}}=0.3 \mathrm{eV}$ and periodicity $p=32 \mu \mathrm{m}$. Fig. 4 (a) shows that with the length $l$ increasing, the absorption bandwidth is expanded, but the absorbance falls inside the band. The design is relatively better when $l=0.75 p$ and $l=0.80 p$ with the bandwidth of $1.22 \mathrm{THz}$ and $1.25 \mathrm{THz}$. From Fig.4 (b), with the increasing of $h_{\mathrm{s}}$, the absorbance decreases at high frequencies in the working band. In Fig.4 (c) with the thickness $d$ of polymer becomes larger, the absorption band moves to lower frequencies and its bandwidth becomes narrower also due to the absorbance decrease at high frequencies when the FP cavity is changed. The absorption bandwidth of $d=25 \mu \mathrm{m}$, $30 \mu \mathrm{m}, 35 \mu \mathrm{m}$ is $1.31 \mathrm{THz}, 1.31 \mathrm{THz}$ and $1.22 \mathrm{THz}$ respectively. To catch the better performance of the absorber, we further compared $d=25 \mu \mathrm{m}$ with $d=30 \mu \mathrm{m}$ by judging the band with absorbance higher than $95 \%$. The results show the bandwidth are 0.60 $\mathrm{THz}$ and $1.01 \mathrm{THz}$ for $d=25 \mu \mathrm{m}$ and $d=30 \mu \mathrm{m}$ respectively. Therefore, a design with $d$ $=30 \mu \mathrm{m}$ is better.

\section{Conclusion}

In summary, we have designed and numerically studied a broadband absorber based on multilayer graphene and silicon strips array. The simulation results indicate that with the combined effect of the FP resonance and the dipole mode oscillation in the silicon strip array interacting with graphene, the structure provides a broadband absorption $(0.73 \mathrm{THz}-1.95 \mathrm{THz})$ with absorbance higher than $90 \%$. It is found that the absorption frequency bandwidth of the absorber can be dynamically tuned by changing the Fermi level of graphene. The length, width and height of the silicon strip also exert an influence on the absorption performance. Because of relatively simple structure with no patterned graphene, the proposed absorber may have potential applications such as band-rejection filters and broadband anti-radiation in $\mathrm{THz}$ range.

\section{References}

[1] D. Saeedkia, A.H. Majedi, S. Safavi-Naeini, et al, Analysis and design of a photoconductive integrated photomixer/antenna for terahertz applications, IEEE J. Quantum. Elect. 41(2005) 234-241.

[2] G.P. Williams, Filling the THz gap - high power sources and applications, Rep. Prog. Phys. 69(2005)301.

[3] S. Fan, Y. He, B.S. Ung, et al, The growth of biomedical terahertz research, J. Phy. D: Appl. Phys. 47(2014)374009.

[4] C. Jansen, S. Wietzke, O. Peters, et al, Terahertz imaging: applications and perspectives, Appl. Opt. 49(2010)48-57.

[5] H.T. Chen, W.J. Padilla, M.J. Cich, et al, A metamaterial solid-state terahertz phase modulator, Nature Photon. 3(2009)148-151.

[6] P. Avouris, Graphene: Electronic and Photonic Properties and Devices, Nano Lett. 10(2016) 4285-4294.

[7] F.H. Koppens, D.E. Chang, F.J. García, Graphene Plasmonics: A Platform for Strong LightMatter Interactions, Nano Lett. 11(2011)3370.

[8] D.C. Wei, Y.Q. Liu, Y. Wang, et al, Synthesis of N-Doped Graphene by Chemical Vapor Deposition and Its Electrical Properties. Nano Lett. 9(2009)1752.

[9] A.Vakil, N. Engheta, Transformation optics using graphene, Sci. 332(2011)1291 - 1294. 
[10] K.I. Bolotin, K.J. Sikes, Z. Jiang, et al, Ultrahigh electron mobility in suspended graphene, Solid State Commun.146(2008)351-355.

[11] A.K. Geim, Science, Graphene: Status and Prospects, Science 324(2009)1530-1534.

[12] K.S. Novoselov, V.I. Fal'Ko, L. Colombo, et al, A roadmap for graphene, Nature 490(2012)192-200.

[13] F.M. Kong, H.W. Zhuang, K. Li, et al, Plasmonic bandpass filter based on graphene nanoribbon, Appl. Opt. 54(2015)2558-64.

[14] A.Vakil, N. Engheta, One-atom-thick reflectors for surface plasmon polariton surface waves on graphene, Opt. Commun. 285(2012)3428-3430.

[15] D. Ansell, I.P. Radko, Z. Han, et al, Hybrid graphene plasmonic waveguide modulators, Nat. Commun. 6(2015)8846.

[16] H. Tao, C. M. Bingham, D. Pilon, et al, A dual band terahertz metamaterial absorber, J. Phy. D: Appl. Phys.43(2010)225102.

[17] M. Janneh, A. D. Marcellis, E. Palange, et al, Design of a metasurface-based dual-band Terahertz perfect absorber with very high Q-factors for sensing applications, Opt. Commun. 416(2018)152-159.

[18] T. Meng, D. Hu, Q. Zhu, Design of a five-band terahertz perfect metamaterial absorber using two resonators, Opt. Commun. 415(2018)151-155.

[19] A. Fallahi, and J. Perruisseau-Carrier, Design of tunable biperiodic graphene metasurfaces, Phys. Rev. B. 86(2012)4608-4619.

[20] A. Andryieuski, A.V. Lavrinenko, Graphene metamaterials based tunable terahertz absorber: effective surface conductivity approach, Opt. Express 21(2013)9144-55.

[21] Y. Zhang, Y. Feng, B. Zhu, et al, Graphene based tunable metamaterial absorber and polarization modulation in terahertz frequency, Opt. Express 22(2014)22743.

[22] H. Xiong, J. Dong, M. C. Tang, et al, Ultra-thin and broadband tunable metamaterial graphene absorber, Opt. Express 26(2018)1681-1688.

[23] M. Rahmanzadeh, A. Abdolali, A. Khavasi, et al., Adopting image theorem for rigorous analysis of a perfect electric conductor-backed array of graphene ribbons, J. Opt. Soc. Am. B 35(2018)1836.

[24] M. Rahmanzadeh, H. Rajabalipanah, A. Abdolali, Multilayer graphene-based metasurfaces: robust design method for extremely broadband, wide-angle, and polarization-insensitive terahertz absorbers, Appl. Opt. 57(2018)959.

[25] X. Zou, G. Zheng, J. Cong, et al, Polarization-insensitive and wide-incident-angle optical absorber with periodically patterned graphene-dielectric arrays, Opt. Lett. 43(2017)46.

[26] Z. Xu, D. Wu, Y. Liu, et al, Design of a Tunable Ultra-Broadband Terahertz Absorber Based on Multiple Layers of Graphene Ribbons, Nanoscale Res Lett. 13(2018)143.

[27] P. Fu, F. Liu, G. J. Ren, et al, A broadband metamaterial absorber based on multi-layer graphene in the terahertz region, Opt. Commun. 417(2018)62-66.

[28] A. Fardoost, F. G. Vanani, A. Amirhosseini, et al, Design of Multi-Layer Graphene Based Ultra Wideband Terahertz Absorber, IEEE Trans. Nanotechnol. 16(2017)68-74.

[29] E.S. Torabi, A. Fallahi, and A. Yahaghi, Evolutionary Optimization of Graphene-Metal Metasurfaces for Tunable Broadband Terahertz Absorption, IEEE Trans. Antennas Propag. 65(2017)1464-1467.

[30] J.T. Kim, C.G. Choi, Graphene-based polymer waveguide polarizer, Opt. Express 20(2012)3556.

[31] L.A. Falkovsky, Optical properties of doped graphene layers, J. Exp. Theor. Phys. 
106(2008)575-580.

[32] Hugen Yan, Tony Low, Wenjuan Zhu, et al. Damping pathways of mid-infrared plasmons in graphene nanostructures, Nat. Photonics. 7(2013) 394-399. 